Nergeid̨ni Des Der neuen Bildergalletie.

Die Fcabemifhe isน Tena

Die seabemies Sattol. in mass burg 7 Exemplace.

Die Acabemies sandl. in Strass burg, 2 Exemplare.

Sr. Earpar Semrid ackens, Raufmann in Reubaus im Bremijœen.

- Búngermeifter albert in San: nover.

- 9 mmon.

- Eellato Mrmé

- Doctor anberion.

Die anbraifde Batiolutis in grantfurt 3 Erempl.

St. Sanileibireftor anbra.

- Benator Anteimann.

- Seinrid von itrnlut.

- v. 'Arrim in Teu, Ieminen.

- Eandrath vor arraim itt Edtoartenfe.

Die 5rn. Artaria und Eomp. in sien.

Die คern. Fudbånblet Fad; mann uno Bunbermann if Samburg, 41 Exempl.

Sr. Tobann Tobias פalter in Dabme.

- Toh. Sarl Eottfi. Saltet in Dabme.

- Raufmann Bater.

- S. 5. Бarbiet.
Şr. Ets. Watdemis in Gdinio Deberg.

Gtau oberforfmeiftetin ฯ. Bar netsig geb. v. Soutenburg.

Bartboloi, Profeffor de: Matur: gefochichte in Etettin.

Sr. Bebeimtatb Baumgarten:

- Sijudbánoler Baumgartter iii Seipitg, 4 Exempl.

Dem. Charlotte Rbeobore Ama lie sederet.

Sr. Budbandlet Bed to Motor lingen, 3 Exetnal:

Den. \$illbelmine $\mathfrak{B e b t e t t o s . ~}$

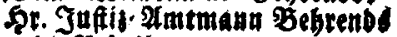
in seituberg.

- Doctor $\mathfrak{3} . \mathfrak{P}$. Dedmant.

- Fudbănoler Beer in Leipig. 3 Eremplare.

- or. Benionelit.

- S. S. Эerg in 2atlatt g Erpl.

- Saufmanin Serget in Ner fabt Eberswalde:

- Bottfies Serget.

- Ebriftian Bottl. Betger.

- Frieor. Eubw. Besto.

- Sammarferr and grótat $s$. Blatentée in Filebule.

- Sob. Ebrif. \$3ilbelm Bler iif Branbettsurs.

一 . א. Blliet.

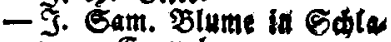
$x:$

5 5. 


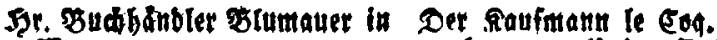

2B.e:.

- Ruboig Rlumberg.

- Eunnibat Boldo tu Ratbenau, 2 Ex.

- Bidbdader Boba in Şams buig, 13 Frentel.

Die Srr. Bobn und Eomp. in Qúbec, 14 Erempl.

Shr. QuDnig von Bobelming.

ftl. Eopbte oon 500 flwing.

Sot. Lurwig Fonte.

Dem. Totanne Marie Ragoas lene $\mathfrak{B}$ ofilingta.

Sr. Friebrto Pbilipp Gebbarb oon FreDor.

- Frielrio Quonis silbelt bon Bredom.

Sr. Majorin von Brebow.

Sr. Mua. Ferotwano Bretfa.

- Reatorungdacootat $\mathfrak{B r t h}$ in Darmiftaot.

- Brod.

- Budbánder Bronner in Frantfut, 3 Ftempl.

- Inipector-Fidie.

Die Brudneriobe Sanbluth in Dantig, 6 Exempl.

Dem. Wilbetmtae Sruber.

Die frt. Brdfin v. Brubl.

Set. Srriedr. Braf yon ฬrutbt.

- Qevporo von Bŭlow in Sets Del.

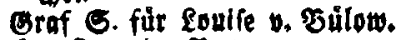

Gr. Eourtier Fußze.

- cabantee.

- Eaubs und Landfofafts : sato o. Eomielesto.

- B. R. Sabs.

- Sunfer Guftas bon Earifien.

- Imterath Safpart.

- opil Elexment.

Der Erbpring o. Eoburg Sodf. Durd lautht.

- Banquter (Soben.

- Peter Andreas Eollomb, in Srantf. a. $\$$ R.

- Sacques le Somte.

- Eebeimrath le Soq.

- Diretiol It Soq.
- Sausoour Cof in Felefeos.

- ঙhauf Erank.

- Budbudndler Éras in Sreys berg.

- כudbander Sreun in sorag: bebura, I Exempl.

Dem. Mar de la Eroit.

Sr. Babbatisler Crutus it Leiptig, 6 Exempt.

- Buthdir Dh Danget in Duff feliorf, 2 Tremol.

- Gohann Salo - on Danfert In Srante a J)?

- Sohnan $D$ void Darfert in Srantfurt a M.

- Predigr Danj in Meufadot 3 Ftemo!are.

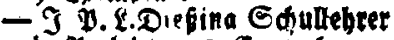
in Porlebera, 8 Exempl.

- Budb)dnoter Dietrid in Góttingen, 3 Exempl.

- Girrift. WBilb. Dippe in Sals berfaot.

Dem. Senriette Dipve.

- Bulbelmine Dippe.

grt. Sentette von Dornberg.

5r. frik von Dỏnberg.

- Eouis von Dórmberg.

- Jobana Gafar Dorge.

- Orrift Driefen, flugel Iojutant $6 r$. Saifert. JRaje fät in Detersbutg.

- Droft boll פundobajer in Sardevieh.

- Dute.

Arizgaratb Drenet.

- Preo. Dürow su Bernilow

- 5. Duffetmalb in SRiefen: burs.

Der $\mathfrak{B}$ irobauer Eben.

Die Srn. Budbandler Ebrbars

uno sofiuns in Stutegaro, 3 Efemplare.

Detti. Seanette Ctøbols.

5ृ. D. Eiфmann, Erbbert auf (sỏfernip, Rierile uno 6 teg: lin.

- 8. 5. Eimte.

Dem. Jobanna Eifelen. 
5r. Prdifbent von Eifenbers.

- Saufimana Epbraim.

- Suc. Erben zu Aitenbof.

- Sr. B. Erma.

- Buctbansl. Ernft in Quebs. Itncurg, 4 Eximpl.

- Budbánoler Eglinger in Srantfutt, 6 Exempl.

- Budabdnoler Ettinger in Gotbar 3 Exempl.

- Duerfarss E Enots.

- Faber in Frantfurt a Expt.

- Jofrach Jalle in Şannoper.

- Eonortor recot.

- I. E. Feiso, Reftor fu Bernan.

Dem. Tobanne Sharlotte fies linus i: Sis onall.

Sr. Fudbbdubier Felsecter in Nitunoera, 6 Exempl.

- rencler in Sosiendorf.

- Semufmana ferting.

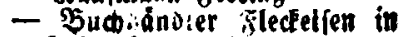
Silmtiout, 2 Exempl.

- Budbáabl. Jieilder in leips f.a, 7 Exen il.

Stau Ben. Rati tn Jlefd a Expl.

Dem. Jobantre Eupurentue deos poldine Ebarlotte fieffow th Berfe/

St. Appotbefer Flitner, \& Enl.

- MRa ifter Flitmer auf Reus bof bei Dúben.

- von Flotmani, Sammer. Rati).

- Major v. Sorcabeauf Solels mik.

- Franion.

Accife : und Bollectetaic Gtans fe in Branoenturg.

Frant Eroptint tu Eoharg, Sooch fürat. Durdblaudt.

Ser. Fudbander Friebt! in libat, to Exempl.

- Gudbanbler frommann in Sülliфau, 14 Exempl.

- ऊudb. Fùdfel in 3eebf 6 Exempi.

- Sufar adolab fati.
Şr. Fuđbănol. Gablet in Yena.

2 Exemplate.

- Staupemana v. Garten, vort Sung: Sow etinidien oregi: ment.

- Piof. Bedile, gu Fauken.

Die Sorr. Budionatler Gebra uub haupt in $\mathfrak{R}$ aroied.

Sr. Barou bon Beift, senanat yoi Barren.

- Seopolb Bented in Gata.

- Tau: Benedi in Slaks.

Dem. Shentipte (senebi inslak. Sp. Bitterldafitsatb won Oets bard.

- Fudhodnder Betlad in oresben, if Exemplate.

- 5. Berlast.

- OIeguft Seriad.

- Emtor Bersborf.

Det Domainet, uno Et. Tos banniter : Maltbejer: Droens. Sammerrath 5r. Biefefe.

Spr. Tranadt Blake in 20 als denburg.

- Referendariud Giefele.

- Bauinípeltor Blafemalo.

- Brteds. Earl sueobor Bosss ner in Barlin.

- Regierungeratb cosing.

- Buinbander Grau in Sofo 6 Eximplare.

Dem. Gat.dhen Grebin.

- Ebar,otte hentiette SBeate Grieb/क.

- Eleonote Griebfón.

SR. Budbander Briedbad in Eaffel.

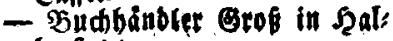
berftad.

- Eena Ebeod. Graber, Goha Des Sgerrn Magiftratsratbs Bruber su Brobi in Ballizien.

Dem. Jobantre Jultane Oüts ther in Blogau.

- Sentiette Búntber.

- Ea:oline Oúntber in 52Rags Debura.

- Marie Ebarlotte Gintbet in Ragbeburg. 
5r. Fubbinbet Sunthet in Slogau, 28 Exempl.

Gt. Dberbofmeif. v. Bunblađ. Sr. Major von Buonneau.

Sr. Sammerzath Outife

- Pammerberr von Saad.

- Simtátaso Saafen auf Fralls endorf.

Dem. Augufte Sanact.

- Satel ja Ganfiouri.

Sct. Bebeim. Finamarato $\mathfrak{B a s}$ ron vpa der jagen,

- Prediger $\mathfrak{5} a b n$.

- Eantor Sabn.

Sercen Gebruber Saßn, Bad. bdul. in Sannov. 40 Exempl.

- Seinr. EBilbelm Soaden in Stargard in Pommern.

- Juffigr. Soracfen du Neuen Damerow,

- Budbander hammeria in Alsora, 5 Exempl.

Dem. Dorothea von Şammers fiein in Rienburg.

Sr. Qubroig \$aron von Satt: merfetin.

Graf 5 , füt Melufine Bargit von Sommerfitin.

Sr. Prebiget - Sarting in $3 \mathrm{~B}_{\mathrm{rg}}$.

Fr. yen Jartmant, geb. ven Stanomsty.

Br. Budodnoler Sart?noch in Riga, 36 Elempl.

D. Sartimann aus magseb.

- Saufm. Sartmann in Sals berfact, 4 Exemplare.

- Holms, Elb' 30l: amt: Affifient.

- Ebrif. Milb. Sartmann in Sagoeburg.

- Bucibandler $\mathfrak{S a r t u n g}$ in $\mathfrak{P}^{2}$ nigsoerg, 29 Exempl.

:- Seinrí v. Seautdarmov.

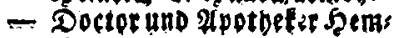
pel.

- Sixufm. Seitmann 3 Exempl.

- Zriebr. ferdin. 23 in vremsitin.

- Budbanoler sellmis in Şannoues! 4 Exemplare.
Shr. Gref uon Sgenfel.

- Saufmann Peter Şentel in Samburo.

Dem. Jobanna Sgenidel.

Sir. Sendler.

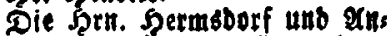
ton in Borlik, 5 . Exemplare. Sr. Auguft goillb. Joertig.

- D. 20. Sildebranbe invotes.

- Infpeftor foildetrand.

- Job. Catl heilíder.

- Iuftii = Imtmann Soermana ii Terbellin.

- Job. Earl Iugnt Siridel.

- Foft Secretatr Hobed ia Şamn 3 Exempl.

- Eorffecret. Shöne in Driefer.

- Iobann Ebriftian Szofmann in Reu: Srandenb. 4 Erempl.

- v. \$poff, 2Augufi friebria.

- Preo. Sodfe in Crobtoten.

- Sucibander 50 fimann in Samburg, 40 Exempl.

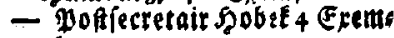
plare.

- Rammerfectet. Fomever.

- E. Finantratb Şonis.

Pem. IImalia Şubemann it Beman.

Die Srrn. Sortin unb Comp. in 5ुern, 4 Exempl.

Br. Jeinr. Friecr. Soutb in grantfutt.

- 厄. 5 . Janid in \$albents buts.

- $\mathfrak{H}$. G. $\mathfrak{H} \cdot \mathfrak{D}$. Sabn in Perleberg.

- T. F. Jeffe, Rỏnigl. Erbs

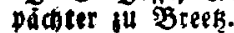

- von Ilten in Seftorf.

Sointgl. Eburfürțl. Intelligents Comt. in Sagnover, 39 Ex.

Das Satelligent : Eomtoir in Leipug, 12 Exemplare.

St. Sfede in Gillebre, a Exems plare.

- gioles und Tatob senjas min 9 kzig.

5f. Dberbaurätbin Ikig.

5r. \$̣enjamin Jateb Jkib. 
St. Futboublet Iberfet.' Dem. Tungbenbel in Berdiet. F̧r. Buhbảudler Raffein Stet: tin, 8 Excmpl.

Frálein v. Siallifa th Berbft. Frau Majorit von Rame.

- Detramtmana Rarbe. SRr. Bubbander gaven in $\mathfrak{A l}$ tona, 1.2 Exempl.

- Ebrenfried Seintid भnguft v. Seffelbrind o. Griebon.

- Bdttl. Midanelis Reibel.

- Frtiedr. 2Billb. Ema sev bel.

- ร. S. 23. Selbel.

Sadame Rerfen.

Serru Reflet uno Sofulte.

- Staberetretair unb Infits Commiffarins Sino uno Qübs befe.

Sr. Ebrif. Gottlieb Piefling, Saufmasn in Siridberg.

- Silimm im Gr. Ríditre.

- J. E. \& Situnt.

- von Sicifi, Eburf. ERd6f. Landidafts: Dikector it Melsborf bet Dabme.

- Xoilbelm oor Rrebel.

- Eecretair sinoblaus.

- Eeorg Frtibrín Sinotbe.

- Sofratb Sod ingettia bel Salle.

Dem. Bilto. Sobnemant.

St. Carl Jeinr. Sot!b. Iuguf ooll Sorigstorf, aus betn Soauie Robernift.

- Friedr. BBilh. yon solse.

- 5udbänder Job. Friebrid Sorn in Stredlan, 27. Exems plare:

- Fuchánblet 23 . O. Potu in Freslau, 73 Exempl.

Die einder bes Serrn $\$$ addtet Inton Staafe in IDenaven. Sor. Sirifit.

Madame la Colonel Comtesse de Krockow.

Shr. Suffiłbürgermeiffer Srodi: fius in Deutíd Erone.

-- Dberforfmeifter son Sropf.
Gr. D. Setratr, in Renfabt C.

- Butbbalter fertaer.

- Ernft Ioesder Benedictus Su்let.

- Ernfi zbeobor seltedictu: Rúblet.

- Sobann Wilb. Sübnel.

- Feintio friebr. Samuel Sumit.

- Sudbandl.fenmmes in Reip. tia, Io Exempl.

- Doct. Sturella.

Tulie nno Qouife Lacanat.

$\mathfrak{S}_{2}$. Preoiger Eampert in $\mathfrak{I n}^{\mathrm{n}}$ ferburg, 4 Exemplare.

Der Geb. Irtbunalsratb Sert yott lampre $\phi t$.

- Landrée.

- Apotbeler lange in Zoffett.

Dem. Carol. Langenmager it Somfebeberg.

Spt. Gerblaund wilb. Eauffer

- Ernit von Rebebur.!

- Dbertadmeifer Bebmants.

- Dem. Rebmann.

for. Fambert. Lemte in Balt.

- Butbr. Lebmann in Rbort. 6 Exemplare.

- 2 Peffer.

- Esullebrer zerfonter in Dberfomiebeberg.

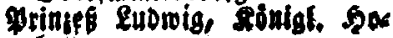
beit.

- Dant. Saeny.

- Ealomer Daniel Loeby.

- Eiesmanar.

Dem. Eardithe Qiez̧mantr.

- Imalia Einbetaana.

5r. vor linfingen.

- Preb. Qlbfo in Branbeub.

- Bublyindl. fơme in Bresl. Gans friedr. Geint. Bottlob'v. Sittowis auf Edsonau.

Ser. Earl Sherrmant, Oraf vod Eottum.

- Obrifi son Lombars geb. b. Sarnemik, 7 Erembt.

- Rauermeiffer Eube.

Srl. Frieberife von Lombow.

- Conbegiane boll Lombon. 


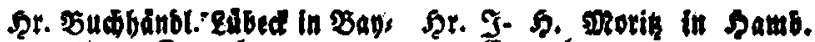
reuth, 3 Exempl.

- Earl Ribete.

- Firreges - und Domainentath Rüematui in Eoslin.

- 2ugufit githersoorf.

- 5̧ans Srreorio von eitts mis in Ectionat.

3 Exemplaze.

- snukentbedier.

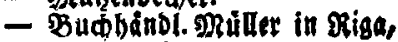
26 Eeempi.

Sri. Oebeimr. Müller, $b$. Erfte.

Dem. Eliatlotte Múller.

- Sudiundier פRăen in Eeer, 2 Exempl.

- von Mantenfel.

- von $\mathfrak{M a r m}$ B.

- von mauberobe.

- von Mrarconnay

- Eudpeig Maurer.

- Evuir Mavet.

- Ecoullebrer. Bu buvorwerf.

- Bet. Ratb Or. Maver.

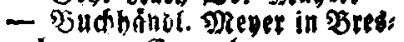
Ina, it Exrmpl.

- Bouch ranor. shener in Lem. go $=$ Ekembr.

- SRengen in samburg.

Dem. vahuelmine Mesger.

Sor. Bucibdnoter Bietler in Stuttgard, 3 Eremul.

Madame Midaast.

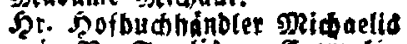
in $\mathfrak{B}_{3}$. Strelis 4 Exempl.

- Io arm Seimrí Bottlieb शidanetis.

- Bottl. Midaelió ia vajes male.

- ubemaøer msalinger.

- Oeb. Tatb Rónid.

Dem. Natie Saroline Soutife Ehanutte Friederife 2 atugu: fine Nollenbauer.

Şr. Momm in Eolberg.

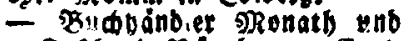

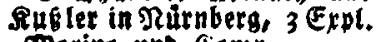

- SRorino uno Comp.

- Budbandiet Montas in 9ie: gensourg, 2 Exempl.

- Mofer, Rodnigl. Doez : Baal. Rath.

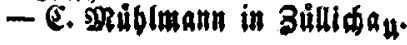

- Emilie $\mathfrak{x}$ Bilbelm. \$üllet.

- Florentine : soilbelm. DR üller.

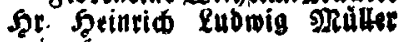
in ḩannover.

Detm. Sentrette פeullet.

Dr. Muller, $\mathfrak{S a u f m}$. in BreAf.

- Gorfimelfer $\mathfrak{R}$ etter in Grof́ ren.

- Geb. Binanzratb Reubaus.

Dem. Guliane Reubaus.

J̧r. enopold Reubaus.

- Obet: Accifes and 3ollratb शitau in Seandenburg a.b. havel.

- Bubbandler Nicolovius in ก̃uigst. 16 Exempl.

Dem. Frtederife Louife Dowas thee Nitfide in Meodibor.

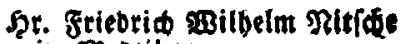
in speosibor.

- O̧minuesmeifarr Dertel.

- Dbréprge in Cottbus.

- Bebeimr. Delriche 2 Expl.

- Ebrift. sBill. auguft Dls Delop in Riga.

- Ebrifi. Fried. Wiltb. Otto.

- Bactbándler Pala in Ea langen, 3 Exempl.

- Sob. Friedri申 Patifius in Eemplin.

- Frteor. Bitl. Jac. Pakturff

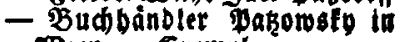
\$Ben, 2 Erempl.

- Sraf uon Podemils.

- 2 Alpbonfe shonod paye.

- Iob. Guberiam Polidinow. Dern. Rariane Pope.

Br. Yoffiestr. Pralle in Celle, 4 Exempl.

- Budbandler Proft uno Stord 
Gtotd) in Copettbagen, 7 Ser. Bubbandrer Ritider in Exemplare.

Şr. Proken in æBuferbaulen.

- Carl seint. Qubr. Bottl.

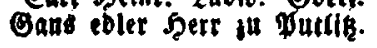

Grl. Aupufte von Putteammer, in Mobrow.

Sp. Eugen ven Puttlammer, in Mobrow.

- Ehedoor voll Puttammet, in Pobraw.

Sil. Emilie von Puttemmet in Pobrom.

- $\mathfrak{R}^{* * *}$ fít $3 \mathfrak{x o ́ d t e r ~ i n ~} \mathfrak{R}$ rienmerber.

Sr. Priegtratb Rade in mas rienburg.

Srl. Selene Egarlotte Elifab. von Gabmel.

Sr. Prebiger Redlic in Quess bilich.

- Rebfelo in Pojeu.

- Eapellmeifter Reicjaro.

- Meimann in Breftan.

- Bubbitubte setnite in Eeiprig, 4 Exempl.

- Jobann Daniel Reinel in Spandau.

Dem. Dorotbee Gopbie Rei nele, in Epanbau.

St. Beb. Ratb Reufuer.

Dem. Infepbe Renuer.

Se. Durdl. ber regier. Giftef, Sheincich Der 33 fte Reub, in Yoberftein.

Sr. Budbindler Ridter in Dresben, 6 Exempl.

- Prebiger $\mathfrak{E}$. Mubolph sid: ter in gr. Biethen.

- Earl Stiebr. खill. Riemann.

- Dber s Felo: Stabosmedicus Ri:m?r.

Şr. Eati Riejenberg. Satnnover, 5 (Exempl.

- Ritter, Eonfui in surtertr walde.

- Canunich 20iff. Rih.

- Earl Friebr. æBilb. Mobrig.

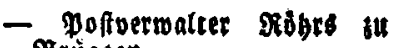
Brüggen.

Fr. Plegiments, Evirurgus Rl: ps ben.

- Maufm. Nobde in Anflam. Ser. Beneral v. Rooid) Excels Ient.

- Mober unb mefel, 30 Ex.

- כaron son Robert.

- Rofmana in Patibor.

- Rotbe uno Eomp. 3 Expl.

- Eenator Ruder, 3 Exenpr.

- von Rable.

- Megiments:Quartiermeifer Rumpler.

Dem Erueft.Şenriette Rüpdef. Şr. Earl Aug. Ferd. Ruppel.

- Sriebrid Ernt Rúfoer in Szannover.

- Eotiog Ealontan.

Die Srrn. Sitauntburg u. Comp. in Bien, 16 Exempl.

Dem. Auguffe Sdeel. 5. von Gdetidan in Botba. Grau Maiorin a. Gdiertiot. Şr. Maurmetfter Sdilste. S2r. Job. Cbrift. Sdiv. Srau Beb. Rátbin v. Stlab: rentorf.

Sr. Eraf von Solabtenborf in Eráben.

- Sanbratb yon Sdoning in Morrn.

- Rriegdratb Sdlúper.

Dem. 
Detu. To5. Cbriff. Sdmibt, Eodter bes Spern Canflets Directors.

Ser. Submig Edmitb.

- Sdnadenbarg, Eanonicus.

- Eanoratly von Sqoining in xorm.

- (Beh). Finangratb Sacm: mer.

- Artbur Sooppenbauer it Şamburg.

Dem. Rouife Milbelm. Sareis bet.

Dem. Pbilippine Ebarlotte Sobreiber.

Sr. Whith. Bottl. Sdreibet.

- Buchbandler Sorteinef in Duffiloorf. 6 Exempl.

SR. Poft:Commiffarius Stro: Der in Marienmerder, $2 \mathcal{E F}$ emplare.

- Sriedr. Saroder.

- Dber: Áccife : mo 30lls ratb Gdrdoer in Frandens burg.

- Fudhandler Saroder in Bralirfortig, 2 Ex: mpl.

- Inlpector Edroder st Pukar.

- Wothermalter Soubart in Bremen, 3 Exempl.

- Sutt $\mathfrak{A}$ malius Sottfried Gchabbert aus lubin bei Gollinom.

- Raufman G中úler.

- Eqube licenteinnebmer in muftron.

Die Stul : Sudbanblung it Braunidroig, 6 Erempl.

Die Bráfin uon Soulenburg.

Jit. Ariegstatb Soluls.

Dem. Sufanne grarie Sळult ill fiff.. a. $\mathfrak{R}$.

- Rebecta Edult.

Str. Y. E. Sdhul in Perleberg.

- Beb. Finantrath v. Sकuli.

- 2loolph SBill. FerD. Sdulke.

- D. $\mathfrak{B}$. Edulie in Etettiu.

- Earl friedr. 2lug. Equlje.

- enufuana Gatife.
Şr. Bòttøermeifter Sळulte iี Grabors.

- Cbriff. Friebr. Soulte in şrieken.

- Jobant Soachim Dieftí Soulie.

- Sudw. Sdumana ju silfe in Detureusen.

Die Frn. Yudbinbler Sđwax uno 80 is in Mannbeim, 6 Exempl.

Sุr. Qubro. Carl Sdmets in altoita.

frăul. Friebetile frevin bon Edwenint in Damboorf.

Sat. Altolab Oraf y. Sotwerit.

- Seliger fen. Sudbunbet in Angermunte.

- Eariley : Director Settin.

- Eieferant Siebert.

- Fudbandler Severin in Bet fenfeld, 2 Exempl.

Deit!. Siebecte.

J)r. Budbåndet Siegert, 12 Erempl.

Dem. Thilipp Gotzmann.

fr. Epliztegarb.

- Eandidat Esponbolf.

- Bubbdrol. Stabel u. Sit lian in Peft, 6 . Exempl.

- Rendant Eteglid, 2 Erems plare.

- von Steinmert.

Srall Eieutn. von Sternfeld in Rienburg.

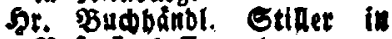
Roftoct, 6 Exempl.

- Carl Friebrid Stodell.

- amtsr. Gtopdelberg.

- Oreoiger Etudenuano in Bellin.

Frál. Dttilie Grieoer. Souife Sbarlotte Emilie von Enoow.

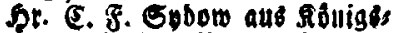
berg in Der Reumart.

Des Drbentsfadutein vor Gys Dow in seboeniaf.

SEr. Baren Sbiele fello. 


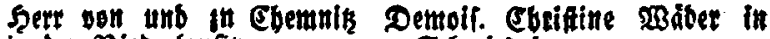
in ber Rieberlaufiz.

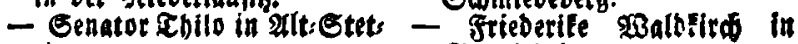
tin.

- Paftor Tbimaus in Fans Ser. Wagner fen. fingbaulen.

Srau yon Sobieffenbaulen in - Baafe, Secret. unb Regis Dablmiks.

Dem. Jobanne Franjigle Cas roline Tbomaderoste in Şeilsberg,

fitl, von ฐbuimen.

ઝ̧. Friebr. Fielte.

- Eriebr. Bilb. Topfet.

- Eúfaermelfter হortilomith in Jnfierburg.

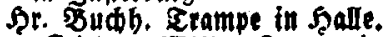

- Friedr. Milth. Ereut in Nauen.

Dem. Frieberife Dorotb. 23ils beimite ?reu.

Dorotbea Elifab. Ereute, geb. Irnolb.

ร.. Э. ह. 2. Lubwig Entuet aus fùrfermalbe.

- Rammer (ectetair stentlet in Bró Blogau.

Sot. Carl Beorge Ebripian Freutler in \$albenburg.

- Budbandler zrofdel in Dantig, 6 Ekempl.

Dem. E. Ern. Souife Truttte.

5r. Fudbainder $\mathfrak{B a n b e n b o e l}$ uno $\mathfrak{R u p r e c t}$ in EDttingen, 6 Exempl.

- Budbandl. Bartentrapp in Granêf. 12 Exempl.

- I. $\mathfrak{E}$. F. Ueberidat aus Nieberfalefien.

- Prä́fibent you Anguabe, 6 Erempl.

- Augafí unbol:

- Beberm. Eecretair Bolfer, 2 Exemplare.

Dim. Soulie Bogel.

Sุp. Braf v. Hktúl Bưlbenbant in Donau. frrator bet Sonigl. Accile: unb 30 LI : Direction in Brans benburg.

- v. Walbow in Mobrentbien.

- Budbandier waltber in Dresben, 2 Exempl.

- Paftor $\mathfrak{B}$ altegott il Blau कe in Sthlepien.

- Sbudbánder maypler in Brien 2 Exempl.

- Etadrato Beidemann.

- Meiomann Ronigl, Eotterie Einnebmer.

- Jr. Meinborb saufmann.

- Ëeb. Eecretá DBeite.

Der Fơnigl. Prin Bulbelnt uon Preusen.

Dem. Ebarl. roilhelm. Elit. Bei

ScP Rummerrath Beibbed.

- Poffecretair Eöblin, 3 Exempl.

- O. Ardivariug אBerniks.

- \$ilb. Weftfelb.

- Tuftir. พ3tchers in Damig.

- Fob. Srieor. SBiegand.

- Drofeffor ख̉ildenom.

Dem. Frieberife $2 B$ ilbelmi.

- Dordbea Wilbelmi.

Şr. Cant $\mathfrak{B i l l e r m a n n . ~}$

- Dostor willía in soittens berg.

- Budbånd. Bilmanns in Bremen, 6 Erempl.

Dent. Wilbelmtne Mintzen.

Dem. Frieberile Juliane Ehars totte Edent v. Winferfiede ju Edimadbauren bei Eelle. SRr. T. E. Đ. Bittte Apotbeler in Sebonict, 2 Exempl. 


\section{$2 \cdot c$}

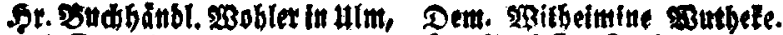

6 Exempl.

Gil. 2 Bubelmine von wolbe in

2Buterwis 1. D. Reumart.

Scr. Sam. Baftbafar $\mathfrak{B}$ olf.

- Seinr. Ebrif. 2301 ff.

- Orora fubw. Jul. Seintio Boifenbaar in Sameln.

5r. Profeifor Benter.

Dem Srieder. Be'dte.

Shr. Tohand Beter Gottfrieb

Bierbotot.

- Bimmermann Sinntbänbles in Sannober.

- Moftmeifter Bimmermann.

- Sormes, Doctor in soltts - Eteglmentsdirurg. Btmmess ftocf. mann.

- arg. 2Billy. yon \$Buffen. - Eecret. J. B. Bombrouner. 